\section{Mildes Asthma ohne Eosinophilie erfordert Behandlungsalternativen}

Lazarus SC et al. Mometasone or Tiotropium in Mild Asthma with a Low Sputum Eosinophil Level. N Engl] Med 2019; 380: 2009 - 2019

Leitlinien empfehlen inhalative Kortikosteroide (ICS) bei allen Patienten mit persistierendem Asthma. Bei Patienten mit mildem Asthma ist aber nicht selten die Eosinophilenzahl im Sputum als Surrogat für eine Entzündung gering. Eine Studie untersuchte, ob eine Therapie mit dem langwirksamen muskarinischen Antagonisten Tiotropium oder dem ICS Mometason bei Patienten mit weniger als $2 \%$ Eosinophilen im Sputum wirksam ist.

Wie die US-amerikanische SIENA-Studiengruppe unter Erstautorenschaft von S.C. Lazarus von der Universität von Kalifornien in San Francisco berichtete, wurden 564 Patienten in einer sechswöchigen Einstiegsphase zunächst unter Placebo charakterisiert, an das Führen eines elektronischen Tagebuchs gewöhnt und bezüglich der Adhärenz untersucht. Anschließend wurden 295 mindestens 12-jährige Patienten mit einem milden, persistierenden Asthma nach dem Eosinophilengehalt (hoch: $\geq 2 \%$, niedrig: $<2 \%$ ) stratifiziert. Jede Gruppe erhielt verblindet im Crossover-Design im Wechsel entweder Mometason und ein Tiotropiumplacebo oder Tiotropium und ein Mometasonplacebo oder zwei Placebos. Die gesamte Studiendauer betrug 42 Wochen. Primärer Endpunkt war das Ansprechen auf Mometason im Vergleich zu Placebo bzw. auf Tiotropium im Vergleich zu Placebo bei Patienten mit niedrigem Eosinophilengehalt im Sputum. Das Ansprechen wurde mithilfe von hierarchischen Endpunkten beurteilt, Kriterien waren Behandlungsversagen, Asthmakontroll-Tage und forciertes Einsekundenvolumen (FEV1). Daneben erfolgte auch ein Vergleich zwischen den Patienten mit hohem und niedrigem Eosinophilenanteil im Sputum.

\section{Ergebnisse}

$73 \%$ der Patienten wiesen in der Eingangsphase der Studie ein niedriges Eosinophilenniveau auf. Alle litten an mehr als zwei Tagen die Woche und mehr als zwei Nächten im Monat an Symptomen oder benötigten mindestens zweimal pro Woche eine Bedarfsmedikation. 59\% dieser Patienten zeigten ein differenzielles Ansprechen auf ein Studienmedikament. Einen signifikanten Unterschied zwischen Mometason und Tiotropium gab es dabei aber nicht: Beim Mometason-PlaceboVergleich sprachen $57 \%$ dieser Patienten auf das ICS besser an und $43 \%$ auf das Placebo. Beim Tiotropium-Placebo-Vergleich war das Ansprechen auf Tiotropium bei $60 \%$ der Patienten besser und auf Placebo bei $40 \%$.

Bei Patienten mit höheren Eosinophilen im Sputum war nur Mometason mit einem signifikant bessern Ansprechen als Placebo assoziiert (74\% vs. $26 \%$ ), nicht aber Tiotropium (57\% vs. $43 \%$ ).

FAZIT

Die Mehrzahl der Patienten mit mildem persistierendem Asthma wies in der Studie geringe Eosinophilenwerte auf. Bei diesen Pateinten erzielten sowohl Tiotropium als auch Mometason ein Ansprechen bei einem vergleichbaren Anteil von Patienten war. Das widerspricht den Leitlinienempfehlungen einer ICS-Dauertherapie, die geeignete Therapie für diese Patienten muss aber erst noch bestimmt werden. Dies sollte nach Meinung der Autoren aber nicht in ICS-kontrollierten Studien erfolgen.

Friederike Klein, München 